\title{
1 Abnormalities of cortical structures in 2 adolescent-onset conduct disorder
}

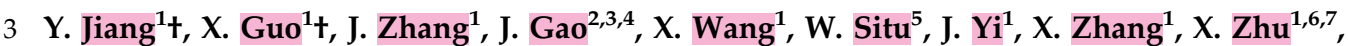 \\ Q1 4 S. Yao ${ }^{1,6,7 *}$ and B. Huang ${ }^{1,8,9 *}$ \\ $5{ }^{1}$ Medical Psychological Institute, the Second Xiangya Hospital, Central South University, Changsha, Hunan, People's Republic of China \\ $6{ }^{2}$ Centre of Buddhist Studies, University of Hong Kong, Hong Kong, People's Republic of China \\ $7{ }^{3}$ Biomedical Engineering Laboratory, Department of Electronic Electrical Engineering, University of Hong Kong, Hong Kong, People's Republic of \\ 8 China \\ $9{ }^{4}$ Alzheimer's Disease Research Network, Strategic Research Theme of Healthy Aging, University of Hong Kong, Hong Kong, People's Republic of \\ 10 China \\ $11{ }^{5}$ Department of Radiology, the Second Xiangya Hospital, Central South University, Changsha, Hunan, People's Republic of China \\ $12{ }^{6}$ National Technology Institute of Psychiatry, Central South University, Changsha, Hunan, People's Republic of China \\ $13{ }^{7}$ Key Laboratory of Psychiatry and Mental Health of Hunan Province, Central South University, Changsha, Hunan, People's Republic of China \\ $14{ }^{8}$ Department of Biomedical Engineering, School of Medicine, Shenzhen University, Shenzhen, Guangdong, People's Republic of China \\ $15{ }^{9}$ Shenzhen Institute of Research and Innovation, University of Hong Kong, Shenzhen, Guangdong, People's Republic of China
}

edition, text revision (DSM-IV-TR). It presents a repeti- 39 tive and persistent pattern of behavior whereby the 40 basic rights of others, or major age-appropriate norms, 41 are violated (APA, 2000). CD has been reported to 42 occur in about $16 \%$ of otherwise healthy preadolescents 43 (Olsson, 2009). According to developmental taxonomic 44 theory (Moffitt et al. 2008), adolescents with early-onset 45 CD (EO-CD) (who exhibited initiated CD symptoms 46 before 10 years) are more susceptible to persistent ag- 47 gressive or antisocial behaviors in their adult life com- 48 pared with adolescent-onset CD (AO-CD) subjects. 49 Correspondingly, the former has been more extensively 50 studied. Although less likely than their EO counterparts 51 to show persistent antisocial problems into young 52 
53 adulthood, AO individuals are more likely to have anti54 social problems and are also expected to experience more health burden in later adult life than their control counterparts (Odgers et al. 2007; Roisman et al. 2010). Some recent evidence also suggests differentiated phenotypic findings as well as task performances, such as reward sensitivity and facial recognition, between AO-CD and EO-CD patients (Fairchild et al. 2009a, b; Passamonti et al. 2010; Silberg et al. 2014). In Fairchild et al. (2011), reduced volume of the right insula was only observed for AO-CD patients compared with healthy controls (HCs), while reduced volume of the amygdala was observed for both subtypes of CD relative to controls. Given the aforementioned findings, we expect to further explore the biological substrate of $\mathrm{AO}-\mathrm{CD}$, thereby providing conceivable evidence for the subtle distinction that may exist between the two subtypes.

To date, several studies have compared the gray matter structure of normally developing youths and adolescents with $\mathrm{CD}$, especially those with EO-CD (Kruesi et al. 2004; Sterzer et al. 2007; Huebner et al. 2008; Fairchild et al. 2011; Hyatt et al. 2012; Wallace et al. 2014). The abnormal structures identified most often included the orbitofrontal cortex (OFC) (Huebner et al. 2008), the amygdala (Huebner et al. 2008; Fairchild et al. 2011; Wallace et al. 2014), the insula (Sterzer et al. 2007; Fahim et al. 2011; Fairchild et al. 2011) and other temporal regions (Huebner et al. 2008; Hyatt et al. 2012). Moreover, gray matter volume in the frontal and temporal areas has often been found to be inversely related to the $\mathrm{CD}$ symptoms manifested by a subject (Sterzer et al. 2007; Huebner et al. 2008). It has been hypothesized that impairment of the aforementioned structures, which may affect emotional regulation and behavioral control (Blair, 2004), is associated with the inappropriate behaviors exhibited by CD subjects (Rubia et al. 2009). Correspondingly, aggressive, antisocial individuals were also found to have structural deficits in the prefrontal cortex, the anterior cingulate cortex (ACC) and several other interconnected regions of the brain (Yang \& Raine, 2009).

Although the structural findings regarding EO-CD have been largely studied in heterogeneous samples and with different study designs, structural alterations of AO-CD have been less investigated and the neural basis of different task performances between the two subtypes of $C D$ remains unknown. In addition, it is important to note that almost all the work conducted before did not exclude attention-deficit/hyperactivity disorder (ADHD) which was characterized by a delay in cortical maturation (Shaw et al. 2007). It indicates that the contribution of co-morbid ADHD features to structural abnormalities observed for $C D$ should be differentiated. Another concern is the use of voxel-based morphometry (VBM); while this 108 method combines both thickness and surface features 109 to calculate gray matter volume (Winkler et al. 2010), 110 it may obscure the degree to which each factor contri- 111 butes to volume differences since these measures were 112 found to be globally and regionally independent and 113 stemmed from different genetic and cellular mechan- 114 isms in the brain (Armstrong et al. 1995; Panizzon 115 et al. 2009). While the surface-based method (surface- 116 based morphometry; SBM) enables separate measure- 117 ment of cortical thickness and surface area as well as 118 cortical folding based on the two-dimensional folded 119 laminar structure of the cerebral cortex (Dale et al. 120 1999; Winkler et al. 2010), it aids in understanding 121 neural abnormalities beyond the basic volumetric ab- 122 normalities and has the potential to elucidate the 123 underlying causes of brain structural alterations and 124 the cognitive processes affected by these abnormalities. 125 In addition, surface-based registration provides signifi- 126 cantly higher accuracy than any form of volume-based 127 registration (Ghosh et al. 2010). Three SBM studies in 128 $\mathrm{CD}$, however, recruited participants with an unspe- 129 cified (Wallace et al. 2014) or wide age range, i.e. 12- 130 18 years in Hyatt et al. (2012) and 16-21 years in 131 Fairchild et al. (2015). Although they matched groups 132 for age, the non-linear and region-specific manner of 133 gray matter development from the ages of 4 to 20134 years may confound group differences (Giedd et al. 135 1999).

In the present study, we decided to set a cut-off of 137 age 14 years for adolescence (Silberg et al. 2014), after 138 the time when puberty has begun in most children 139 and after the patterns of genetic influences have mainly 140 stabilized (Silberg et al. 2001). Additionally, onset of 141 CD after 16 years is rare (APA, 2013), so AO-CD 142 patients aged 14-16 years were recruited along with 143 age-, intelligence quotient (IQ)- and gender-matched 144 HCs. Based on previous literature, we hypothesized 145 that cortical deficits (including thickness, surface area 146 and cortical folding) would be observed in AO-CD 147 patients, especially in the paralimbic regions as has 148 been postulated (Rubia, 2011). Second, the detected 149 cortical deficits were assumed to be correlated with 150 the high-level impulsive as well as antisocial problems 151 in $\mathrm{CD}$.

\section{Method}

Samples

A total of 28 AO-CD participants aged 14-16 years (22 155 males and six females) were recruited from out-patient 156 clinics affiliated with the Second Xiangya Hospital of 157 Central South University (Changsha, Hunan, China). 158 A diagnosis of $\mathrm{CD}$ was determined using the 159 
160 Structured Clinical Interview for DSM-IV-TR Axis I 161 Disorders-Patient Edition (SCID-I/P) (First et al. 2002) 162 by two well-trained psychiatrists. According to 163 DSM-IV-TR (APA, 2000), subjects fulfilling the criteria 164 for AO-CD exhibited at least one symptom of CD after 165 the age of 10 years. To improve the reliability of the 166 diagnostic interview, information was collected from 167 each participant and at least one corresponding parent. 168 A psychiatrist made the final decision if the informa169 tion offered was inconsistent.

170 A HC group was randomly selected from local mid-

171 dle schools in the same region. The HC group was also

172 subjected to the SCID-I/P by the same group of psy-

173 chiatrists that evaluated the $\mathrm{CD}$ group. None of the

174 HCs met the criteria for CD or any other psychiatric

175 disorders, or had history of CD symptoms and aggres-

176 sion. Finally, 30 age-, gender- and IQ-matched indivi-

177 duals (21 males and nine females) comprised the HC

178 group (Table 1), with the Chinese version of the

179 Wechsler Intelligence Scale for Children (C-WISC)

180 (Gong \& Cai, 1993) as the IQ measurement.

181 Participants were excluded based on: a history of 182 ADHD, oppositional defiant disorder, any psychiatric 183 or emotional disorder, diagnosis of any pervasive devel184 opmental or chronic neurological disorder, Tourette's 185 syndrome, post-traumatic stress disorder, obsessive186 compulsive disorder, persistent headaches, head 187 trauma, alcohol or substance abuse over the past year, 188 contraindications to magnetic resonance imaging 189 (MRI), or an IQ $\leqslant 80$ on the C-WISC. Participants 190 were also required to be right-handed, according to 191 the Edinburgh Handedness Inventory (Oldfield, 1971).

192 This study was approved by each school's adminis193 tration and the Ethics Committee of the Second 194 Xiangya Hospital of Central South University. All sub195 jects and their parents were informed of the purpose of 196 this study and written informed consent of all of them 197 was obtained.

\section{Self-report assessments}

199 All participants underwent the Chinese versions of the 200 Center for Epidemiologic Studies Depression Scale 201 (Radloff, 1977) and the Multidimensional Anxiety 202 Scale for Children (Yao et al. 2007b). These scales 203 were used to assess depression and anxiety severity, 204 respectively. In addition, the Chinese version of the 205 Subjective Socioeconomics Status Scale (SSS) (Hu 206 et al. 2012) was used to quantify each participant's 207 socio-economic status, the Strengths and Difficulties 208 Questionnaire (SDQ) (Yao et al. 2009) was used to de209 tect internalization and externalization of problems. 210 Callous-unemotional (CU) traits were evaluated 211 using the Antisocial Process Screening Device (APSD) 212 (Frick, 2001), and the Barratt Impulsiveness Scale
Table 1. Demographics and psychiatric characteristics of adolescents with $\mathrm{CD}$ and $\mathrm{HCs}$

\begin{tabular}{llll}
\hline & \multicolumn{3}{l}{ CD } \\
& HCs & patients & $p$ \\
\hline Demographics & & & \\
$\quad$ Age, years & $15.1(0.6)$ & $14.8(0.8)$ & 0.13 \\
Gender, $n$ & & & 0.46 \\
$\quad$ Male & 21 & 22 & \\
$\quad$ Female & 9 & 6 & \\
C-WISC & $107.4(6.9)$ & $103.3(9.1)$ & 0.15 \\
SSS & $6.2(1.3)$ & $6.1(1.6)$ & 0.82 \\
Psychiatric characteristics & & & \\
MASC & $38.3(12.6)$ & $44.6(20.5)$ & 0.17 \\
CES-D & $12.7(6.9)$ & $14.6(6.6)$ & 0.27 \\
APSD total & $11.0(2.8)$ & $15.3(4.3)$ & $<0.001^{* *}$ \\
APSD-callous- & $4.5(1.5)$ & $6.4(1.8)$ & $<0.001^{* *}$ \\
unemotional & & & \\
APSD-impulsivity & $3.5(2.0)$ & $4.7(2.0)$ & $0.04^{*}$ \\
BIS total & $67.4(8.7)$ & $78.5(12.6)$ & $<0.001^{* *}$ \\
BIS-non-planning & $27.4(4.9)$ & $32.0(5.1)$ & $0.001^{* *}$ \\
impulsivity & & & \\
BIS-attention & $17.8(3.0)$ & $19.1(4.5)$ & 0.19 \\
impulsivity & & & \\
BIS-motor impulsivity & $22.2(3.4)$ & $27.4(5.2)$ & $<0.001^{* *}$ \\
SDQ total & $12.5(5.1)$ & $15.5(5.7)$ & $0.04^{*}$ \\
SDQ-conduct problem & $2.4(1.3)$ & $3.7(1.8)$ & $0.004^{* *}$ \\
\hline
\end{tabular}

Data are given as mean (standard deviation) unless otherwise indicated.

$\mathrm{CD}$, Conduct disorder; HCs, healthy controls; C-WISC, Chinese Wechsler Intelligence Scale for Children; SSS, Subjective Socioeconomic Status Scale; MASC,

Multidimensional Anxiety Scale for Children; CES-D, Center for Epidemiologic Studies Depression Scale; APSD,

Antisocial Process Screening Device; BIS, Barratt

Impulsiveness Scale; SDQ, Strengths and Difficulties Questionnaire.

$$
{ }^{*} p<0.05,{ }^{* *} p<0.01 \text {. }
$$

(BIS) (Yao et al. 2007a) was used to assess impulsive- 213 ness. All CD subjects were treatment-naive. Details 214 regarding psychiatric assessments for the two groups 215 are provided in Table 1.

\section{Image acquisition}

Three-dimensional (3D) T1-weighted images (Philips, 218 Achieva, 3.0T, the Netherlands) for all participants 219 were obtained using 3D turbo field echo sequence. 220 Scan parameters are: repetition time $=8.5 \mathrm{~ms}$, echo 221 time $=3.743 \mathrm{~ms}$, flip angle $=8^{\circ}$, matrix $=256 \times 256$ pix- 222 els, field of view $=256 \times 256$, number of slices $=180,223$ slice thickness $=1 \mathrm{~mm}$, image voxel size $=1.0 \times 1.0 \times 224$ $1.0 \mathrm{~mm}^{3}$, and acquisition time $=178 \mathrm{~s}$. 


\section{Image processing}

All participants' T1 images underwent a radiological evaluation performed by a specialist (W.S.) to assess the presence of abnormal radiological or structural features. No participants were excluded from further analysis because of motion artifacts. Anatomic reconstruction of the cortical surfaces was performed using the Freesurfer image analysis suite (stable release version 5.3.0; http:// surfer.nmr.mgh.harvard.edu) as previously described (Dale et al. 1999; Fischl et al. 1999). Triangle meshes which represent the boundary of the white surface (the gray matter-white matter interface) and the boundary of the pial surface (the gray matter-cerebrospinal fluid interface) were generated using deformation algorithms based on local intensity values (Dale et al. 1999) and geometrical and topological constraints (Fischl et al. 2001). The estimated white and pial surfaces were manually corrected for inconsistencies by visual inspection by an operator blind to each subject's diagnosis. The reconstruction procedure was repeated until accurate representations of white and pial surfaces were obtained. The reconstructed surfaces were used to calculate cortical thickness and surface area (Fischl et al. 1999), with the former estimated as the shortest distance in millimeters between the two surfaces. As a result, cortical thickness values with submillimeter accuracy were obtained from over 100000 vertices per hemisphere.

Estimates of surface area (the total area of the surface encompassing a brain region) are quantified by assigning an area to each vertex equal to the average of its surrounding triangles (Winkler et al. 2012). The total vertex area is summed over all vertices, and it is equal to the sum of the areas of the triangles. The degree of cortical folding (assessed by local gyrification index; 1GI) was measured using surface-based, 3D gyrification measurements according to Schaer et al. (2008), a validated method embedded in Freesurfer. The lGI at a given point on the cortical surface was computed as the ratio between the surface of a $25-\mathrm{mm}$ radius circular region of interest (ROI) on the folded pial surface and the surface of the corresponding cortex's outer perimeter (Schaer et al. 2008). The amount of cortical folding (IGI) at each pial surface location reflects the amount of cortex buried within the sulcal folds in the surrounding area. As correct IGI values are typically between 1 and 5 , the greater the value of the $1 G I$, the more surfaces are buried in sulcal folds (Schaer et al. 2012).

\section{Statistical analysis}

Cortical thickness was smoothed with $10 \mathrm{~mm}$ while the IGI and surface area were smoothed using $5 \mathrm{~mm}$ fullwidth/half-maximum Gaussian kernels. To assess regional between-group differences in these cortical structural measures, surface-based group analyses 279 were performed using the general linear model tools 280 available in Freesurfer. Prior to the group comparisons, 281 each participant's data were resampled into an average 282 spherical surface representation that optimally aligned 283 the sulcal and gyral features across the subjects 284 (Wismueller et al. 1999). Statistically significant differ- 285 ences between the cortical thickness, surface area and 286 IGI of the two groups were identified using a Monte 287 Carlo simulation (Hagler et al. 2006), a cluster-wise cor- 288 rection applied for multiple comparisons. Clusters 289 were initially obtained using a $p<0.05$ (two-tailed) 290 vertex-wise threshold, and these were only reported 291 if they met an additional cluster-wise probability 292 ( $\left.p_{\text {cluster}}\right)$ of $p<0.05$ (two-tailed) at least. Statistically 293 significant clusters with cortical thinning were 294 defined as ROIs, then we mapped those ROIs to all 295 of the individual subjects to extract statistical values 296 for later correlation analyses.

\section{Post-hoc analysis}

To investigate gender and group effects, the two 299 groups were compared using analysis of covariance 300 with the structural thickness used as the dependent 301 variable, and group and gender used as fixed factors. 302 To determine whether potential confounders such as 303 age, IQ, anxiety and depression level influenced the 304 results obtained, a group comparison of thickness 305 was performed by adding each of these factors as a 306 covariate.

Pearson correlation analysis was applied to the BIS 308 or ASPSD total with ROI thickness in all participants 309 and in AO-CD patients, while subscales of interest (in- 310 cluding BIS-motor impulsivity, APSD-CU and 311 APSD-impulsivity) were only used in patients. All cor- 312 relation results were reported if they were associated 313 with a $p$ value $<0.05$, uncorrected.

\section{Results}

Table 1 lists the demographics and psychiatric charac- 317 teristics for both groups. No significant differences in 318 age, IQ, socio-economic status, anxiety or depression 319 were observed between the two groups. However, 320 CD patients had an overall higher total score and sub- 321 scale scores for the APSD, BIS and SDQ compared with 322 the HCs.

\section{Cortical thickness}

There were no group differences in mean cortical thick- 325 ness in either the left or right hemisphere. In the left 326 hemisphere, decreased cortical thickness was associated 327 
Table 2. Clusters of cortical thinning in adolescents in the two hemispheres (HCs $>C D$ )

\begin{tabular}{lrrrrrll}
\hline Cluster number & Max & Size, $\mathrm{mm}^{2}$ & TalX & TalY & TalZ & Number of vertices & Annotation \\
\hline Left hemisphere & & & & & & & \\
1 & -4.0 & 899.1 & -13.9 & -28.0 & 46.3 & 2113 & PCC, precuneus, paracentral \\
2 & -2.7 & 630.4 & -18.1 & 35.4 & -18.7 & 988 & lOFC \\
3 & -2.5 & 590.1 & -12.9 & -96.8 & 14.5 & 759 & Lateral occipital \\
$\begin{array}{l}\text { Right hemisphere } \\
1\end{array}$ & & & & & & & \\
2 & -4.0 & 1298.8 & 65.2 & -17.0 & 3.0 & 3074 & Superior temporal, supramarginal, insula \\
2 & -3.7 & 839.1 & 31.0 & -41.1 & -9.0 & 1385 & Parahippocampal, lingual, fusiform
\end{tabular}

HCs, Healthy controls; CD, conduct disorder; Max, $\log _{10}$ ( $p$ value); Tal (X, Y, Z), Talairach $(X, Y, Z)$; PCC, posterior cingulate cortex; lOFC, lateral orbitofrontal cortex.

with three clusters (Table 2, Fig. 1a-c). The first cluster included the precuneus, posterior cingulate cortex $(\mathrm{PCC})$ and the paracentral area $\left(p_{\text {cluster }}=0.004\right)$. The second cluster was the lateral orbitofrontal cortex (lOFC) ( $\left.p_{\text {cluster }}=0.002\right)$, while the third cluster was the lateral occipital cortex $\left(p_{\text {cluster }}=0.003\right)$. In the right hemisphere, cortical thinning was associated with two clusters (Table 2, Fig. $1 d$ and e). One cluster consisted of the superior temporal cortex, the supramarginal cortex and a small part of the insula $\left(p_{\text {cluster }}=0.0001\right)$, while the other cluster included the fusiform and the lingual/parahippocampal gyrus ( $\left.p_{\text {cluster }}=0.0002\right)$. Each of these clusters survived multiple comparisons ( $p<0.01$, corrected). None of the clusters had greater cortical thickness in CD than HC subjects.

\section{IGI}

A reduced 1GI was observed at the right rostral ACC $\left(p_{\text {cluster }}=0.0002\right)$, and this extended to the medial OFC (mOFC) and the superior frontal cortex in CD patients compared with the HCs (Table 3, Fig. 2). In contrast, the 1GI for the right precentral cortex ( $p_{\text {cluster- }}$ $=0.0001$ ), extended to the postcentral and supramarginal areas, was greater in the CD patients compared with the HCs. Both clusters survived multiple comparisons $(p<0.01$, corrected).

\section{Surface area}

Compared with HCs, CD patients revealed diminished area in two clusters of the right hemisphere; one was the inferior temporal cortex $(p<0.0048)$ which extended to the parahippocampal gyrus and fusiform, while the other cluster included the precentral and the caudal-middle-frontal cortex $(p<0.0088)$. Both clusters survived multiple correction $(p<0.05)$. However, when 1 a more conservative threshold was used $(p<0.01)$, no group difference was found to exist (see online Supplementary Fig. S6 and Table S1).
Potential confounders and correlation of ROIs with self-reported measurements

There was no evidence that gender influenced the 366 results obtained ( $p>0.2$ in the five clusters). Group dif- 367 ferences in cortical thickness for each cluster remained 368 significant after controlling for age, IQ, anxiety and de- 369 pression $(p<0.001)$. $\quad 370$

For all the participants, four out of five clusters were 371 negatively correlated with the APSD (except the right 372 fusiform) and BIS (except the left precuneus) total 373 scores, but only the left lOFC survived multiple com- 374 parisons $\quad(r=-0.44 /-0.43, \quad$ respectively, $\quad p<0.01,375$ Bonferroni, corrected). 376

For the AO-CD group only, APSD-CU was negative- 377 ly correlated with thickness of the right superior tem- 378 poral cortex $(r=-0.4, p=0.04)$ and the right fusiform $(r=-0.63, \quad p<0.05$, Bonferroni, corrected) while 380 BIS-motor impulsivity was inversely correlated with 381 the thickness of the right fusiform $(r=-0.38, p<0.05) 382$ and left IOFC $(r=-0.35, p=0.09)$. In addition, 383 APSD-impulsivity was inversely correlated with 384 lOFC thickness $(r=-0.33, p=0.07)$ with marginal sign- 385 ificance. All correlation figures of CD patients are pre- 386 sented in the online Supplementary materials 387 (Supplementary Figs S1-S5). We found no significant 388 correlations between the total scores of BIS or APSD 389 with ROI thickness.

\section{Discussion}

To our knowledge, this study is the first to document 392 cortical abnormalities in a moderate cohort of AO-CD 393 patients. The results clearly demonstrated that 394 AO-CD was related to cortical thinning in multiple 395 brain regions. As Rubia et al. (2011) previously postu- 396 lated that abnormal activation of the 'hot' paralimbic 397 system, which mediates the control of emotion and 398 motivation (Blair, 2004), was specifically associated 399 with CD (Rubia, 2011), cortical deficits in the left 400 



Fig. 1. Differences in cerebral cortical thickness between adolescent-onset conduct disorder (AO-CD) patients $(n=28)$ and matched healthy controls (HCs; $n=30)$. Images of the left and right hemispheres for each group are presented. Medial $(a)$, inferior $(b)$ and posterior $(c)$ views of the left inflated cerebral surfaces show differences in cortical thickness between the two groups. Lateral $(d)$ and inferior $(e)$ views of the right inflated cerebral surfaces show differences in cortical thickness between the two groups. Colored regions are used to indicate significant differences in cortical thickness between the two groups, with blue representing a greater thickness for the HC group compared with the AO-CD group. The value of the color bar is a $\log _{10}(p$ value). Cluster labels correspond with those provided in Table 2. PCC, Posterior cingulate cortex; OFC, orbitofrontal cortex. 
Table 3. Clusters of gyrification deficits in adolescents in the right hemisphere

\begin{tabular}{lccccccc}
\hline Cluster number & Max & Size, $\mathrm{mm}^{2}$ & TalX & TalY & TalZ & Number of vertices & Annotation \\
\hline$(\mathrm{HCs}>\mathrm{CD})$ & & & & & & & \\
1 & -3.7 & 3622.4 & 8.3 & 37.0 & -3.9 & 5927 & rACC, mOFC, superior frontal \\
$(\mathrm{CD}>\mathrm{HC})$ & 4.0 & 4765.7 & 27.7 & -14.4 & 60.2 & 11449 & Precentral, postcentral, supramarginal \\
2 & & & & & & &
\end{tabular}

Max, $\log _{10}$ ( $p$ value), the positive value (4.0) of cluster 2 represents a converse result of the contrast; Tal $(\mathrm{X}, \mathrm{Y}, \mathrm{Z}$ ), Talairach $(\mathrm{X}, \mathrm{Y}, \mathrm{Z})$; HCs, healthy controls; $\mathrm{CD}$, conduct disorder; $\mathrm{rACC}$, rostral anterior cingulate cortex; mOFC, medial orbitofrontal cortex.

401 OFC, right ACC, superior temporal and parahippo402 campal gyri and the insula in our AO-CD patients 403 closely matched the cortical topography of this system 404 and were consistent with volumetric reductions repeat405 edly identified in studies of subjects with EO-CD 406 (Kruesi et al. 2004; Sterzer et al. 2007; Huebner et al. 407 2008; Fairchild et al. 2011). Thus, deficits in the paralim408 bic system may reflect a non-specific effect of both sub409 types of CD. Moreover, thickness deficits in the 410 paracentral cortex, fusiform and occipital areas were 411 also reported in previous studies on CD (Fairchild 412 et al. 2011; Hyatt et al. 2012). However, our study sug413 gested that exceptional gray matter reductions occur in 414 the left parietal regions with $\mathrm{AO}-\mathrm{CD}$, including the 415 PCC, precuneus and supramarginal gyri which all 416 have not been observed in EO-CD (Kruesi et al. 2004; 417 Sterzer et al. 2007; Huebner et al. 2008; Fairchild et al. 418 2011). The results indicated that gray matter matur419 ation or processes related to these areas have been dis420 turbed due to AO-CD, although the parietal areas have 421 not traditionally been considered major sites of patho422 logical change in CD.

423 Cortical thinning of the precuneus, PCC and supra424 marginal gyri was in line with two recent structural 425 studies of non-co-morbid CD (Hyatt et al. 2012; 426 Wallace et al. 2014), and was also consistent with ab427 normal activation during inhibitory tasks, passive 428 avoidance learning and risky tasks (Rubia et al. 2008, 429 2009; Finger et al. 2011; Dalwani et al. 2014). 430 However, previous structural studies of EO-CD did 431 not detect deficits in these areas (Kruesi et al. 2004; 432 Sterzer et al. 2007; Huebner et al. 2008), and it might 433 reflect that these deficits are specific features of Q5 434 AO-CD. Although the structural imaging study in 435 which volume differences between the two subgroups 436 were compared did not detect parietal differences 437 (Fairchild et al. 2011), perhaps due to the co-morbidity 438 of ADHD in their CD samples, adolescents with 439 EO-CD are more likely to be co-morbid with ADHD 440 than their AO-CD counterparts (APA, 2013). 441 Otherwise, differences can be attributed to different methods adopted (VBM v. SBM). Indeed, activation 442 of the PCC and precuneus has been primarily asso- 443 ciated with various self-referential processes through 444 its interconnection with other midline structures in 445 the brain, including the ACC and OFC (Northoff \& 446 Bermpohl, 2004); thus, abnormalities in these intercon- 447 nected regions could undermine the self-reflection in 448 subjects with AO-CD. Individuals who lack the cap- 449 acity to reflect on the negative consequences of im- 450 moral behaviors would become predisposed to 451 rule-breaking antisocial behavior (Raine \& Yang, 452 2006). However, this assumption needs to be 453 confirmed by further functional MRI studies in which 454 non-self/self-referential processes would be investi- 455 gated among the two subtypes. Alternatively, studies 456 of EO-CD failed to detect deficits in the parietal regions 457 partially due to the dramatically dynamic changes that 458 occur from childhood to adolescence in this area; 459 namely, a subtle decline in the parietal areas in 460 EO-CD patients, if present, could be compensated by 461 an age-related increase in gray matter from childhood 462 to early adolescence (Giedd et al. 1999; Shaw et al. 463 2008). These two alternative options could be exam- 464 ined by combining both structural and functional neu- 465 roimaging data with a longitudinal method. Taken 466 together, these results suggest that the deficits of the 467 PCC/precuneus may be a potential distinctive feature 468 of AO-CD.

Cortical thinning of paralimbic structures, including 470 the OFC, superior temporal gyrus, insula and parahip- $471 \mathrm{Q} 3$ pocampal gyrus, which were closely interconnected, 472 has been consistent with previously identified struc- 473 tural reductions associated with CD subjects versus 474 controls (Kruesi et al. 2004; Sterzer et al. 2007; 475 Huebner et al. 2008; Fairchild et al. 2011; Hyatt et al. 476 2012; Wallace et al. 2014). Yet, some of these studies 477 did not detect deficits in all of these areas (Kruesi 478 et al. 2004; Sterzer et al. 2007; Wallace et al. 2014); poten- 479 tial explanation might rely on the heterogeneity of the 480 samples, such as co-morbidity, age and IQ, etc. The 481 OFC has been shown to play a crucial role in social 482 


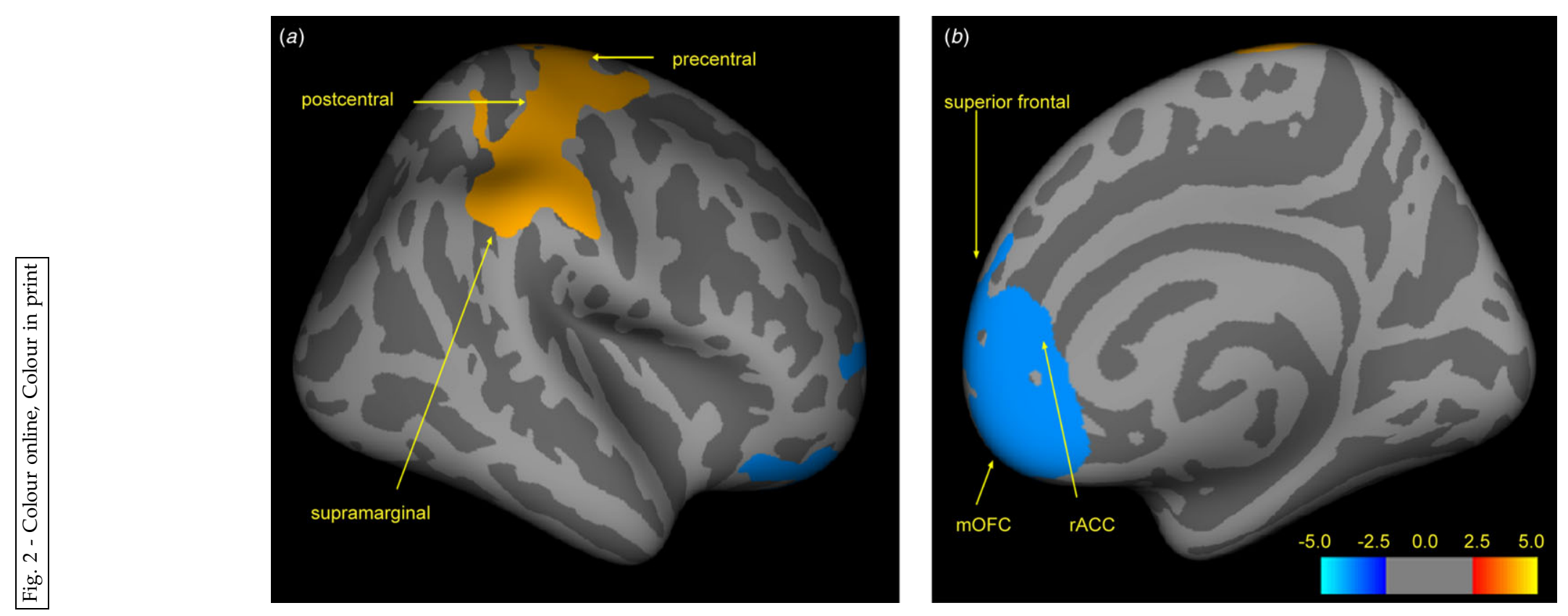

Fig. 2. Cerebral cortical folding differences in the right hemisphere between adolescent-onset conduct disorder (AO-CD) patients $(n=28)$ and matched healthy controls (HCs; $n=30)$. Lateral $(a)$ and medial $(b)$ views of the right cerebral surfaces show differences in the gyrification index between the two groups. Colored regions are used to indicate significant differences in the gyrification index values for the two groups, with blue representing greater values for the HC group compared with the AO-CD group. Conversely, red/yellow coloring represents greater values for the AO-CD group compared with the HC group. The value of the color bar is a $\log _{10}(p$ value). Cluster labels correspond with those provided in Table 3. mOFC, Medial orbitofrontal cortex; rACC, rostral anterior cingulate cortex. 
cognition (Blair \& Cipolotti, 2000), reward and punishment processing (O'Doherty et al. 2001; O'Doherty, 2004); abnormalities in these processes have been closely related to aggression, which is a major characteristic of CD (Blair, 2004). Convergent evidence from functional MRI has also suggested lower activation of the OFC in response to reward task and emotional stimuli processing in $\mathrm{CD}$ adolescents compared with controls (Herpertz et al. 2008; Rubia et al. 2009). Abnormalities in the insula (Sterzer et al. 2007; Fairchild et al. 2011, 2015) have been associated with lack of empathy, and may contribute to abnormal emotional processing among $\mathrm{CD}$ subjects. Cortical deficits in the right superior temporal cortex and fusiform gyri found in our and previous studies (De Brito et al. 2009; Fairchild et al. 2011; Hyatt et al. 2012; Wallace et al. 2014) have the potential to explain why facial expression recognition was impaired in both EO-CD and AO-CD subjects (Fairchild et al. 2009a). The right fusiform gyrus maintains facial expression recognition probably through its communication with the superior temporal cortex (Winston et al. 2004) and OFC (Hornak et al. 2003). Thus, cortical thinning of these structures may compromise the understanding of others' feelings and intentions, leading to a perception of ambiguous social cues as threatening (Fairchild et al. 2008). Together, we speculated that deficits in these paralimbic structures reflect a non-specific effect of $C D$ and play a crucial role in the development of $C D$.

Abnormal IGI values detected in the right ACC were in line with $\mathrm{lGI}$ and volume alterations in CD (De Brito et al. 2009; Hyatt et al. 2012). The ACC plays an essential role in controlling responses (Bush et al. 2000) since the structural and functional organization of the ACC ideally enables it to participate in willed motor control via its extensive connections with the prefrontal cortex (Paus et al. 1993) and the precentral cortex (Dum \& Strick, 1991). Deficits in the ACC have been found in CD and aggressive adolescents (Sterzer et al. 2005; Stadler et al. 2007; Gavita et al. 2012; Hyatt et al. 2012), especially on the right side (Boes et al. 2008).

However, the increased lGI of the precentral cortex seems inconsistent with the results of Hyatt et al. (2012) and Wallace et al. (2014), and the discrepancies could be attributed to the heterogeneity of the subjects (AO-CD only $v . \mathrm{CD}$, and age distribution) or the interactions between genes and environment, since gyrification which was largely determined genetically has also been shown to experience developmental alterations that occur from childhood to adolescence (White et al. 2010), as the microstructure of neuronal sheets (Richman et al. 1975) and axonal connectivity (Van Essen, 1997) have all been shown to affect cortical folding. Aberrant higher-order structures, like the ACC and $\mathrm{OFC}$, together with lower-order motor regions, like the precentral cortex, may undermine the con- 538 trol-motor circuit, thereby resulting in poor regulation 539 of impulsive behavior. $\quad 540$

In general, gyrification is also thought to be intrinsic- 541 ally related to surface area (Eyler et al. 2011), but the 542 diminished surface areas detected in the right inferior 543 temporal and the precentral cortex in the present 544 study were only partly overlapped with areas with 545 folding alterations. Of note, the reduction of surface 546 area $(p<0.05$, corrected) was not as robust as altera- 547 tions of gyrification $(p<0.01$, corrected). Surface area 548 is known to be associated with both number of cortical 549 folds (i.e. local gyrification) and separation between 550 cortical folds (i.e. sulci) (Frye et al. 2010). A discrepancy 551 between alterations in surface area and folding in CD 552 patients, for example (Wallace et al. 2014), may be 553 due to an illness-related disproportional development 554 of the brain gyri and sulci (Casanova et al. 2010; 555 Shokouhi et al. 2012), and this assumption needs to 556 be addressed in future. 557

The robust negative relationship between impulsive 558 or antisocial symptoms and the thickness of the lOFC 559 irrespective of diagnosis implies that impulsive and 560 antisocial behavior is closely associated with cortical 561 thinning in this region. The IOFC plays a pivotal role 562 in top-down control (Elliott \& Deakin, 2005), and defic- 563 its of the IOFC might be a shared neural substrate 564 underlying impulsivity and antisocial behaviors 565 (Blair, 2004), rather than a specific feature of a certain 566 mental disorder. $\quad 567$

While in AO-CD patients only the negative correla- 568 tions between $\mathrm{CU}$ and the thickness of the fusiform, in- 569 cluding the lingual and parahippocampal gyri came out 570 with significance, which was consistent with Fairchild 571 et al. (2015). This implies the close relationship between 572 the $\mathrm{CU}$ traits and processes maintained by the above 573 structures, such as facial expression recognition 574 (Winston et al. 2004). We found no statistically signifi- 575 cant correlations between BIS-motor impulsivity (or 576 APSD-impulsivity) and cortical thickness, but both of 577 them indicated a similar negative trend. Thus, our 578 results demonstrated that cortical thinning in these 579 areas, such as the OFC, fusiform and parahippocampal 580 gyrus, was associated with a higher level of impulsivity. $581 \mathrm{Q} 3$

Interestingly, although we ruled out co-morbidity 582 such as ADHD, ODD, etc., the results of the present 583 study are largely consistent with those of previous 584 studies. This is not uncommon in brain imaging stud- 585 ies, since a meta-analysis (on more than 20000 subjects 586 and 26 different brain disorders) showing that MRI 587 lesions that were common across all brain disorders 588 were more likely to be located in hubs of the normal 589 brain connectome (Crossley et al. 2014). According to 590 'graph theory' (van den Heuvel \& Sporns, 2011), struc- 591 tural deficiencies, including the OFC, ACC, superior 592 
593 temporal cortex, insula, PCC, and precuneus in the 594 present study, match well with the 'hubs' of cerebral 595 cortex which play a pivotal role in attracting and inte596 grating neuronal information across the whole brain. It 597 may lead to a conclusion that the high-value hubs of 598 human brain networks are more likely to be anatomic599 ally abnormal than non-hubs in many (if not all) brain 600 disorders. However, the triggering of a certain brain 601 disorder may rely on complex relationships of the 602 whole brain, including the architecture, neurotransmit603 ters of the brain and so on, and this needs to be inves604 tigated in future studies.

\section{Limitations}

606 There were potential limitations in the present study. 607 First, the cross-sectional nature of the present study 608 constrained us from inferring whether the structural 609 abnormalities observed in the present AO-CD cohort 610 are caused by latter triggering of multiple structures, 611 or represent an abnormal developmental trajectory of 612 these structures, and, as DSM-5 pointed out, AO-CD 613 individuals are less likely to persist into adulthood 614 compared with those with EO-CD (APA, 2013); so, 615 whether the observed deficits were limited in adoles616 cence also needs to be answered. Longitudinal obser617 vation will enable us to uncover the developmental 618 emergence of cortical markers of AO-CD, thereby help619 ing us to identify those who are at high risk of devel620 oping such disorder and seeking protective factors 621 that can delay or even prevent the onset of CD. 622 Second, we did not include EO-CD samples in the pre623 sent study, and so it remains unknown whether the 624 observed AO-CD specific deficits reflect distinct patho625 physiological processes or the heterogeneity of poten626 tial confounding variables in our samples compared 627 with previous EO-CD samples. Nevertheless, given 628 the relative abundance of evidence on EO-CD, it is 629 still reasonable to conclude that our study initiated a 630 valuable insight into this question. Therefore, to better 631 understand the neural basis of CD with respect to age 632 of onset, future work examining the brain structural 633 features of both subtypes of CD in multi-center and lar634 ger samples is needed.

\section{Conclusion}

636 In summary, structural abnormalities identified in this 637 AO-CD cohort are similar to those previously observed 638 for EO-CD, except for the parietal cortex. Thus it is 639 possible that the PCC/precuneus deficits identified in 640 the present AO-CD cohort provide valuable insight 641 into a potential distinction between the two subtypes 642 of CD, despite their shared features. Importantly, in 643 contrary to Moffitt's original notion, these and previous results suggest that the etiology of both sub- 644 types share a biological vulnerability (Silberg et al. 645 2014), and they reinforce a possibly quantitative, rather 646 than qualitative, distinction between the etiology of the 647 different onset of CD (Fairchild et al. 2013). Following 648 this line of reasoning, our study provides supportive 649 evidence for the revision of this theory. However, fur- 650 ther studies are needed to better address this issue.

\section{Supplementary material}

For supplementary material accompanying this paper 653 visit http://dx.doi.org/10.1017/S0033291715001361

\section{Acknowledgements}

This work was supported by grants from the National 656 Key Technologies R\&D Program in China's 11th 657 5-year plan (grant number 2009BAI77B02); the Natural 658 Science Foundation of China (grant numbers 81471384659 and 81370034); and the Specialized Research Fund for 660 the Doctoral Program of Higher Education (grant num- 661 ber 20130162110043), as well as the construct program 662 of the key discipline in Hunan province (S.Y.).

None.

\section{References}

APA (2000). Diagnostic and Statistical Manual of Mental Disorders: DSM-IV-TR. American Psychiatric Publishing: Washington, DC.

APA (2013). Diagnostic and Statistical Manual of Mental 668 Disorders: DSM-5. American Psychiatric Publishing: Washington, DC.

Armstrong E, Schleicher A, Omran H, Curtis M, Zilles K 673 (1995). The ontogeny of human gyrification. Cerebral Cortex 674 5, 56-63.

Blair RJ (2004). The roles of orbital frontal cortex in the 676 modulation of antisocial behavior. Brain and Cognition 55, 677 198-208.

Blair RJ, Cipolotti L (2000). Impaired social response reversal. 679 A case of 'acquired sociopathy'. Brain 123, 1122-1141. 680

Boes AD, Tranel D, Anderson SW, Nopoulos P (2008). Right 681 anterior cingulate: a neuroanatomical correlate of 682 aggression and defiance in boys. Behavioral Neuroscience 122, 683 677-684.

Bush G, Luu P, Posner MI (2000). Cognitive and emotional influences in anterior cingulate cortex. Trends in Cognitive Science 4, 215-222.

Casanova MF, El-Baz AS, Giedd J, Rumsey JM, Switala AE 688 (2010). Increased white matter gyral depth in dyslexia: 689 implications for corticocortical connectivity. Journal of 690 Autism and Developmental Disorders 40, 21-29. 
Crossley NA, Mechelli A, Scott J, Carletti F, Fox PT, McGuire P, Bullmore ET (2014). The hubs of the human connectome are generally implicated in the anatomy of brain disorders. Brain 137, 2382-2395.

Dale AM, Fischl B, Sereno MI (1999). Cortical surface-based analysis. I. Segmentation and surface reconstruction. Neurolmage 9, 179-194.

Dalwani MS, Tregellas JR, Andrews-Hanna JR, Mikulich-Gilbertson SK, Raymond KM, Banich MT, Crowley TJ, Sakai JT (2014). Default mode network activity in male adolescents with conduct and substance use disorder. Drug and Alcohol Dependence 134, 242-250.

De Brito SA, Mechelli A, Wilke M, Laurens KR, Jones AP, Barker GJ, Hodgins S, Viding E (2009). Size matters: increased grey matter in boys with conduct problems and callous-unemotional traits. Brain 132, 843-852.

Dum RP, Strick PL (1991). The origin of corticospinal projections from the premotor areas in the frontal lobe. Journal of Neuroscience 11, 667-689.

Elliott R, Deakin B (2005). Role of the orbitofrontal cortex in reinforcement processing and inhibitory control: evidence from functional magnetic resonance imaging studies in healthy human subjects. International Review of Neurobiology 65, 89-116.

Eyler LT, Prom-Wormley E, Panizzon MS, Kaup AR, Fennema-Notestine C, Neale MC, Jernigan TL, Fischl B, Franz CE, Lyons MJ, Grant M, Stevens A, Pacheco J, Perry ME, Schmitt JE, Seidman LJ, Thermenos HW, Tsuang MT, Chen CH, Thompson WK, Jak A, Dale AM, Kremen WS (2011). Genetic and environmental contributions to regional cortical surface area in humans: a magnetic resonance imaging twin study. Cerebral Cortex 21, 2313-2321.

Fahim C, He Y, Yoon U, Chen J, Evans A, Pérusse D (2011). Neuroanatomy of childhood disruptive behavior disorders. Aggressive Behavior 37, 326-337.

Fairchild G, Passamonti L, Hurford G, Hagan CC, von dem Hagen EA, van Goozen SH, Goodyer IM, Calder AJ (2011). Brain structure abnormalities in early-onset and adolescent-onset conduct disorder. American Journal of Psychiatry 168, 624-633.

Fairchild G, Toschi N, Hagan CC, Goodyer IM, Calder AJ, Passamonti L (2015). Cortical thickness, surface area, and folding alterations in male youths with conduct disorder and varying levels of callous-unemotional traits. NeuroImage: Clinical 8, 253-260.

Fairchild G, van Goozen SH, Calder AJ, Goodyer IM (2013). Research review: evaluating and reformulating the developmental taxonomic theory of antisocial behaviour. Journal of Child Psychology and Psychiatry, and Allied Disciplines 54, 924-940.

\section{airchild G, Van Goozen SH, Stollery SJ, Goodyer IM} (2008). Fear conditioning and affective modulation of the startle reflex in male adolescents with early-onset or adolescence-onset conduct disorder and healthy control subjects. Biological Psychiatry 63, 279-285.

Fairchild G, Van Goozen SHM, Calder AJ, Stollery SJ, Goodyer IM (2009a). Deficits in facial expression recognition in male adolescents with early-onset or adolescence-onset conduct disorder. Journal of Child 751

Psychology and Psychiatry, and Allied Disciplines 50, 627-636. 752

Fairchild G, van Goozen SHM, Stollery SJ, Aitken MRF, 753 Savage J, Moore SC, Goodyer IM (2009b). Decision making 754 and executive function in male adolescents with early-onset 755 or adolescence-onset conduct disorder and control subjects. 756 Biological Psychiatry 66, 162-168. 757

Finger EC, Marsh AA, Blair KS, Reid ME, Sims C, Ng P, 758 Pine DS, Blair RJ (2011). Disrupted reinforcement signaling 759 in the orbitofrontal cortex and caudate in youths with $\quad 760$ conduct disorder or oppositional defiant disorder and a 761 high level of psychopathic traits. American Journal of 762 Psychiatry 168, 152-162. 763

First M, Spitzer R, Gibbon M, Williams J (2002). Structured 764 Clinical Interview for DSM-IV-TR Axis I Disorders-Patient 765 Edition (SCID-I/P, 11/2002 revision). New York State 766 Psychiatric Institute: New York. $\quad 767$

Fischl B, Liu A, Dale AM (2001). Automated manifold 768 surgery: constructing geometrically accurate and 769 topologically correct models of the human cerebral cortex. 770 IEEE Transactions on Medical Imaging 20, 70-80. 771

Fischl B, Sereno MI, Dale AM (1999). Cortical surface-based 772 analysis. II: inflation, flattening, and a surface-based 773 coordinate system. NeuroImage 9, 195-207. 774

Frick PJ, Hare RD (2001). Antisocial Process Screening Device: 775 APSD. Multi-Health Systems: Toronto. $\quad 776$

Frye RE, Liederman J, Malmberg B, McLean J, Strickland D, 777 Beauchamp MS (2010). Surface area accounts for the 778 relation of gray matter volume to reading-related skills and 779 history of dyslexia. Cerebral Cortex 20, 2625-2635. 780

Gavita OA, Capris D, Bolno J, David D (2012). Anterior 781 cingulate cortex findings in child disruptive behavior $\quad 782$ disorders. A meta-analysis. Aggression and Violent Behavior 783 17, 507-513.

Ghosh SS, Kakunoori S, Augustinack J, Nieto-Castanon A, 785 Kovelman I, Gaab N, Christodoulou JA, Triantafyllou C, 786 Gabrieli JDE, Fischl B (2010). Evaluating the validity of 787 volume-based and surface-based brain image registration 788 for developmental cognitive neuroscience studies in $\quad 789$ children 4 to 11 years of age. NeuroImage 53, 85-93. 790

Giedd JN, Blumenthal J, Jeffries NO, Castellanos FX, Liu H, 791 Zijdenbos A, Paus T, Evans AC, Rapoport JL (1999). Brain 792 development during childhood and adolescence: a 793 longitudinal MRI study. Nature Neuroscience 2, 861-863. 794

Gong YX, Cai TS (1993). Wechsler Intelligence Scale for 795 Children, Chinese Revision (C-WISC). Map Press: Hunan, 796 China.

Hagler DJ, Saygin AP, Sereno MI (2006). Smoothing and 798 cluster thresholding for cortical surface-based group $\quad 799$ analysis of fMRI data. NeuroImage 33, 1093-1103. 800

Herpertz SC, Huebner T, Marx I, Vloet TD, Fink GR, 801 Stoecker T, Shah NJ, Konrad K, Herpertz-Dahlmann B 802 (2008). Emotional processing in male adolescents with 803 childhood-onset conduct disorder. Journal of Child 804 Psychology and Psychiatry, and Allied Disciplines 49, 781-791. 805

Hornak J, Bramham J, Rolls ET, Morris RG, O'Doherty J, 806 Bullock PR, Polkey CE (2003). Changes in emotion after 807 circumscribed surgical lesions of the orbitofrontal and 808 cingulate cortices. Brain 126, 1691-1712. 
Hu M, Wang M, Cai L, Zhu X, Yao S (2012). Development of subjective socioeconomic status scale for Chinese adolescents. Chinese Journal of Clinical Psychology 20, 155-161.

Huebner T, Vloet TD, Marx I, Konrad K, Fink GR, Herpertz SC, Herpertz-Dahlmann B (2008). Morphometric brain abnormalities in boys with conduct disorder. Journal of the American Academy of Child and Adolescent Psychiatry 47, 540-547.

Hyatt CJ, Haney-Caron E, Stevens MC (2012). Cortical thickness and folding deficits in conduct-disordered adolescents. Biological Psychiatry 72, 207-214.

\section{Kruesi MJ, Casanova MF, Mannheim G, Johnson-Bilder A} (2004). Reduced temporal lobe volume in early onset conduct disorder. Psychiatry Research 132, 1-11.

Moffitt TE, Arseneault L, Jaffee SR, Kim-Cohen J, Koenen KC, Odgers CL, Slutske WS, Viding E (2008). Research review: DSM-V conduct disorder: research needs for an evidence base. Journal of Child Psychology and Psychiatry, and Allied Disciplines 49, 3-33.

Northoff G, Bermpohl F (2004). Cortical midline structures and the self. Trends in Cognitive Science 8, 102-107.

Odgers CL, Caspi A, Broadbent JM, Dickson N, Hancox RJ, Harrington H, Poulton R, Sears MR, Thomson WM, Moffitt TE (2007). Prediction of differential adult health burden by conduct problem subtypes in males. Archives of General Psychiatry 64, 476-484.

O'Doherty J, Kringelbach ML, Rolls ET, Hornak J, Andrews C (2001). Abstract reward and punishment representations in the human orbitofrontal cortex. Nature Neuroscience 4, 95-102.

O'Doherty JP (2004). Reward representations and reward-related learning in the human brain: insights from neuroimaging. Current Opinion in Neurobiology 14, 769-776.

Oldfield RC (1971). The assessment and analysis of handedness: the Edinburgh Inventory. Neuropsychologia 9, 97-113.

Olsson M (2009). DSM diagnosis of conduct disorder (CD) - a review. Nordic Journal of Psychiatry 63, 102-112.

Panizzon MS, Fennema-Notestine C, Eyler LT, Jernigan TL, Prom-Wormley E, Neale M, Jacobson K, Lyons MJ, Grant MD, Franz CE, Xian H, Tsuang M, Fischl B, Seidman L, Dale A, Kremen WS (2009). Distinct genetic influences on cortical surface area and cortical thickness. Cerebral Cortex 19, 2728-2735.

Passamonti L, Fairchild G, Goodyer IM, Hurford G, Hagan CC, Rowe JB, Calder AJ (2010). Neural abnormalities in early-onset and adolescence-onset conduct disorder. Archives of General Psychiatry 67, 729-738.

\section{Paus T, Petrides M, Evans AC, Meyer E (1993). Role of the} human anterior cingulate cortex in the control of oculomotor, manual, and speech responses: a positron emission tomography study. Journal of Neurophysiology 70, 453-469.

Radloff LS (1977). The CES-D scale: a self report depression scale for research in the general population. Applied Psychological Measurement 1, 385-401.

Raine A, Yang Y (2006). Neural foundations to moral reasoning and antisocial behavior. Social Cognitive and Affective Neuroscience 1, 203-213.
Richman DP, Stewart RM, Hutchinson JW, Caviness VS Jr 869 (1975). Mechanical model of brain convolutional 870 development. Science 189, 18-21. 871

Roisman GI, Monahan KC, Campbell SB, Steinberg L, 872 Cauffman E; National Institute of Child Health and Human 873 Development Early Child Care Research Network (2010). Is 874 adolescence-onset antisocial behavior developmentally $\quad 875$ normative? Development and Psychopathology 22, 876 295-311.

Rubia K (2011). "Cool” inferior frontostriatal dysfunction in 878 attention-deficit/hyperactivity disorder versus "hot" 879 ventromedial orbitofrontal-limbic dysfunction in conduct $\quad 880$ disorder: a review. Biological Psychiatry 69, e69-e87. 881

Rubia K, Halari R, Smith AB, Mohammed M, Scott S, 882 Giampietro V, Taylor E, Brammer MJ (2008). Dissociated 883 functional brain abnormalities of inhibition in boys with $\quad 884$ pure conduct disorder and in boys with pure attention $\quad 885$ deficit hyperactivity disorder. American Journal of Psychiatry 886 165, 889-897. 887

Rubia K, Smith AB, Halari R, Matsukura F, Mohammad M, 888 Taylor E, Brammer MJ (2009). Disorder-specific 889 dissociation of orbitofrontal dysfunction in boys with pure 890 conduct disorder during reward and ventrolateral $\quad 891$ prefrontal dysfunction in boys with pure ADHD during 892 sustained attention. American Journal of Psychiatry 166, 893 83-94.

Schaer M, Cuadra MB, Schmansky N, Fischl B, Thiran JP, Eliez $S$ (2012). How to measure cortical folding from MR images: a step-by-step tutorial to compute local gyrification index. Journal of Visualized Experiments: JoVE e3417.

Schaer M, Cuadra MB, Tamarit L, Lazeyras F, Eliez S, Thiran JP (2008). A surface-based approach to quantify local cortical gyrification. IEEE Transactions on Medical Imaging 27, 161-170.

Shaw P, Eckstrand K, Sharp W, Blumenthal J, Lerch JP, Greenstein D, Clasen L, Evans A, Giedd J, Rapoport JL (2007). Attention-deficit/hyperactivity disorder is characterized by a delay in cortical maturation. Proceedings of the National Academy of Sciences of the United States of America 104, 19649-19654.

Shaw P, Kabani NJ, Lerch JP, Eckstrand K, Lenroot R, Gogtay N, Greenstein D, Clasen L, Evans A, Rapoport JL, Giedd JN, Wise SP (2008). Neurodevelopmental trajectories of the human cerebral cortex. Journal of Neuroscience 28, 3586-3594. Changes in the sulcal size associated with autism spectrum 915 disorder revealed by sulcal morphometry. Autism Research 916 5, 245-252.

Silberg J, Moore AA, Rutter M (2014). Age of onset and the 918 subclassification of conduct/dissocial disorder. Journal of 919 Child Psychology and Psychiatry, and Allied Disciplines 56, 920 826-833.

Silberg JL, Rutter M, Eaves L (2001). Genetic and 922 environmental influences on the temporal association 923 between earlier anxiety and later depression in girls (vol 49, 924 pg 1040, 2001). Biological Psychiatry 50, 393-393. 925 Stadler C, Sterzer P, Schmeck K, Krebs A, Kleinschmidt A, 926 Poustka F (2007). Reduced anterior cingulate activation in 927 
aggressive children and adolescents during affective stimulation: association with temperament traits. Journal of Psychiatric Research 41, 410-417.

Sterzer P, Stadler C, Krebs A, Kleinschmidt A, Poustka F (2005). Abnormal neural responses to emotional visual stimuli in adolescents with conduct disorder. Biological Psychiatry 57, 7-15.

Sterzer P, Stadler C, Poustka F, Kleinschmidt A (2007). A structural neural deficit in adolescents with conduct disorder and its association with lack of empathy. NeuroImage 37, 335-342.

van den Heuvel MP, Sporns O (2011). Rich-club organization of the human connectome. Journal of Neuroscience 31, 15775-15786.

Van Essen DC (1997). A tension-based theory of morphogenesis and compact wiring in the central nervous system. Nature 385, 313-318.

Wallace GL, White S, Robustelli B, Sinclair S, Hwang S, Martin A, Blair R (2014). Cortical and subcortical abnormalities in youths with conduct disorder and elevated callous unemotional traits. Journal of the American Academy of Child and Adolescent Psychiatry 53, 456-465.

White T, Su S, Schmidt M, Kao CY, Sapiro G (2010). The development of gyrification in childhood and adolescence. Brain and Cognition 72, 36-45.

Winkler AM, Kochunov P, Blangero J, Almasy L, Zilles K, Fox PT, Duggirala R, Glahn DC (2010). Cortical thickness or grey matter volume? The importance of selecting the phenotype for imaging genetics studies. NeuroImage 53, 1135-1146.

Winkler AM, Sabuncu MR, Yeo BT, Fischl B, Greve DN, Kochunov P, Nichols TE, Blangero J, Glahn DC (2012).
Measuring and comparing brain cortical surface area and other areal quantities. NeuroImage 61, 1428-1443.

Winston JS, Henson RN, Fine-Goulden MR, Dolan RJ (2004). fMRI-adaptation reveals dissociable neural representations of identity and expression in face perception. Journal of Neurophysiology 92, 1830-1839.

Wismueller A, Vietze F, Dersch DR, Leinsinger GL, Ritter H, Hahn K (1999). Adaptive self-organized template matching of the gray-level feature space for automatic segmentation of multispectral MRI data of the human brain. Radiology 213, 364-364.

Yang Y, Raine A (2009). Prefrontal structural and functional brain imaging findings in antisocial, violent, and psychopathic individuals: a meta-analysis. Psychiatry Research 174, 81-88.

Yao S, Yang H, Zhu X, Auerbach RP, Abela JR, Pulleyblank RW, Tong X (2007a). An examination of the psychometric properties of the Chinese version of the Barratt Impulsiveness Scale, 11th version in a sample of Chinese adolescents. Perceptual and Motor Skills 104, 1169-1182.

Yao S, Zhang C, Zhu X, Jing X, McWhinnie CM, Abela JR (2009). Measuring adolescent psychopathology: psychometric properties of the Self-Report Strengths and Difficulties Questionnaire in a sample of Chinese adolescents. Journal of Adolescent Health 45, 55-62.

Yao S, Zou T, Zhu X, Abela JR, Auerbach RP, Tong X (2007b). Reliability and validity of the Chinese version of the Multidimensional Anxiety Scale for children among Chinese secondary school students. Child Psychiatry and Human Development 38, 1-16. 